\title{
Optical and RF power requirements for a new injection-locked semiconductor laser diode method compared with conventional approaches for QPSK and QAM modulation
}

\author{
R. Slavík ${ }^{(1)}$, B. Kelly ${ }^{(2)}$, R. Phelan ${ }^{(2)}$, J. O'Carroll ${ }^{(2)}$, and D.J. Richardson ${ }^{(1)}$ \\ 1. Optoelectronics Research Centre, University of Southampton, Highfield Campus, SO17 1BJ, \\ Southampton, UK, r.slavik@soton.ac.uk \\ 2. Eblana Photonics Ltd., Unit 32 Trinity Technology and Ent. Campus, Pearse St., Dublin 2, Ireland
}

\begin{abstract}
Our recently-developed QAM synthesis using direct modulation of injection locked semiconductor lasers is analyzed in terms of the optical and RF power requirements and compared with IQ modulation and multiple binary modulator schemes.
\end{abstract}

\section{Introduction}

Nested Mach-Zehnder IQ electro-optic modulators have been a key device in the development of coherent optical communication systems. However, when generating higher order modulation formats (16 QAM and beyond), multilevel high-speed data streams are required (e.g., 16 QAM requires I and Q RF streams both formed by 4 -amplitude level signals). Generating these multilevel signals is challenging, especially at high speeds, and requires drivers capable of delivering high RF power while also maintaining low distortion in terms of linearity. It has been suggested that this issue can be mitigated through the use of more complicated modulators with multiple electrical inputs and multiplexing the signals in the optical rather than electric domain [1]. Recently, we presented another solution that goes one step further, where in addition to performing multiplexing in the optical domain, it completely avoids the use of external modulators, even when QAM signals are to be generated [1]. The scheme is based on ultralow chirp direct modulation of injectionlocked (IL) semiconductor lasers followed by coherent addition and carrier suppression of their output. Using this scheme, operation has been recently [3] demonstrated at baud rates up to 24 Gbaud, with formats up to 16 QAM and operation over a wavelength range of $30 \mathrm{~nm}$ in the C-band [3].

Here, we analyze and attempt to compare all three techniques (IQ modulator, multipleintegrated modulators and our new scheme) from the point of view of both optical and RF power requirements, allowing a critical comparison of these three approaches for the first time.

\section{Principle of operation}

Initially we describe how our new scheme works and follow this with a brief description of the other methods.
Fig. 1a shows the operating principle for the simplest modulation format; BPSK. We first injection-lock a directly-modulated laser by feeding a component of $\mathrm{CW}$ light from a master laser into a current-modulated slave laser. If both lasers emit at the same wavelength, they become phase locked, meaning that the slave frequency follows that of the master, even in the presence of the slave laser modulaton. This is an established method to suppress the chirp of directly-modulated lasers [4]. Moreover, as the slave laser output is phase-locked to the master, stable interference between them is possible. Consequently, the carrier of the amplitude modulated slave laser can be eliminated by interfering it destructively with an appropriate portion of the $\mathrm{CW}$ master laser light, producing BPSK modulation as illustrated in Fig. 1a [2,3].
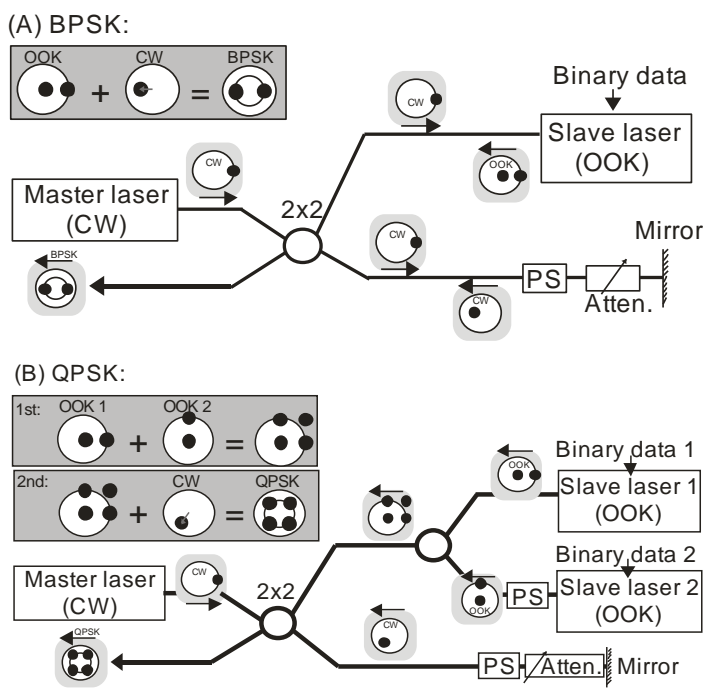

Fig. 1: Principle of operation of our direct scheme.

This can be simply extended to QPSK as shown in Fig. 1b. Here, we modulate two lasers with two binary data streams, both of them injectionlocked to the same master. As the two modulated lasers are phase locked, we can combine them with a 90 degree optical phase shift, producing a four-level modulation that 
resembles QPSK, except for the fact that it contains the carrier. Similar to the way BPSK signals can be synthesized, the carrier is suppressed through destructive interference with a CW component from the master, as illustrated in Fig. 1b. We can scale this further as an example later we show 16 QAM synthesis using four binary-data modulated slave lasers.

Now, let us return to the comparison of the three schemes, the schematics for which are shown in Fig. 2. We consider push-pull driving of all modulators - an IQ modulator is shown in Fig. $2 \mathrm{a}$ - it requires two independent data streams representing the $I$ and $Q$ of the data signal. In Fig. $2 b$ [1] we see a configuration in which each modulator is fed by a single binary data stream. For QPSK, it is identical to an IQ modulator, but for 16 QAM, it requires four modulators, each producing one BPSK signal. Our scheme is schematically shown in Fig. 2c - as compared to the scheme shown in Fig. $2 \mathrm{~b}$, it has directlymodulated lasers instead of modulators and one additional arm that provides the CW master laser signal for the carrier suppression. Similarly to the previous scheme, it uses only binarymodulated data streams. However, it requires only one single-ended RF data stream per laser, as it does not operate in a push-pull mode.

The constellation diagrams in Fig. 2 show in detail how QPSK and 16 QAM are generated. For the IQ modulator, QPSK requires two binary data streams that are combined coherently at the Mach-Zehnder output coupler. For 16 QAM, I and $Q$ each consist of a four-level RF electrical signal, Fig. 2a-iii. For the second modulation scheme, Fig. 2b, 16 QAM can be viewed as a coherent summation of two QPSK signals (each of them produced by two modulators in a similar way that QPSK was generated using the IQ modulator), Fig. 2b-iii. In our scheme, only amplitude-based signals are generated carrying one bit of information each, so the carrier has to be finally removed via destructive interference. The processes for BPSK, QPSK and 16 QAM are outlined in detail in Figs 2c-ii,iii,iv. The optical powers given in the constellation diagrams will be explained in the next section.

\section{Power analysis}

\section{Optical loss}

Here, we neglect the propagation loss and consider only loss due to splitting and interference, which allows us to compare different schemes without considering fabrication challenges/difficulties. For simplicity, we calculate all the characteristics for set input powers (e.g., laser power) and put the resulting numbers directly into Fig. 2. All the lasers considered here emit a peak power of $1 \mathrm{~mW}$ (in CW lasers, this corresponds to an average power of $1 \mathrm{~mW}$ ). We calculate two parameters for each scheme: scheme loss and modulated power. The former indicates how much generated optical power is lost (here, we consider only optical power generated by the signaling lasers) and modulated power is the output power at the output of the device. The results are summarized in Table 1. In obtaining these numbers we considered optimum splitting of the input coupler in the scheme shown in Fig. $2 b$ of $40 / 40 / 10 / 10$.

\section{(a) IQ modulator}

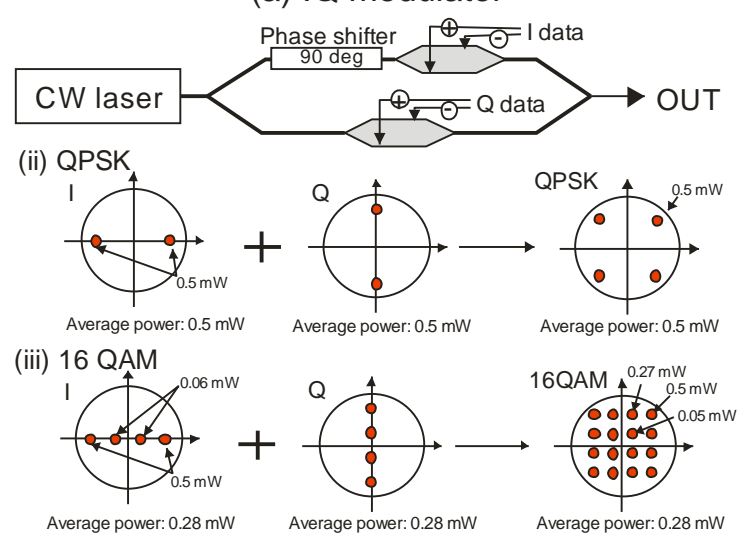

(b) Optically-multiplexing modulator

(i) Schematics

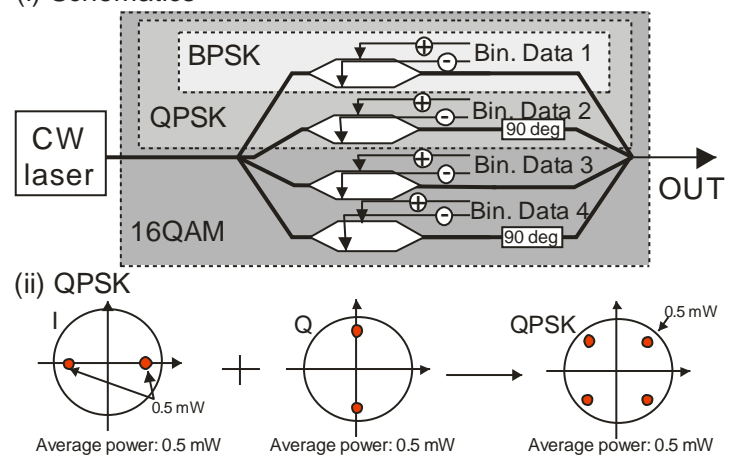

(iii) 16 QAM

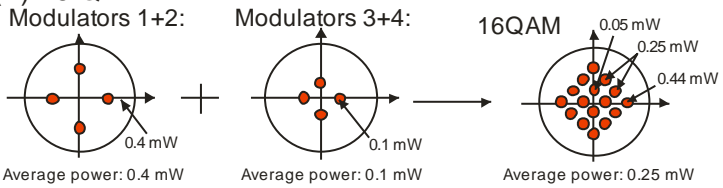

(c) Our scheme

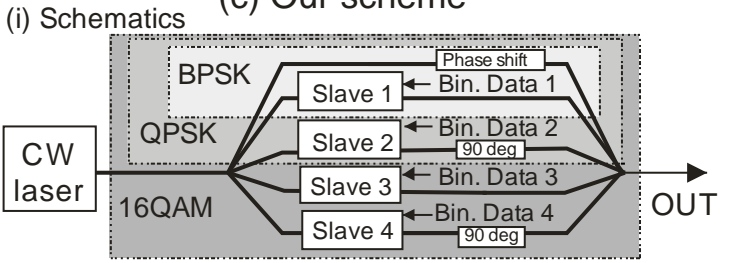

(ii) BPSK

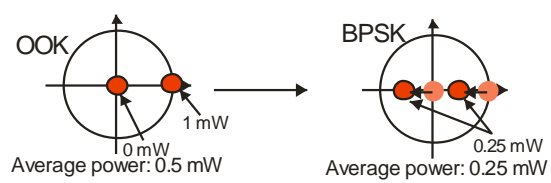


(iii) QPSK

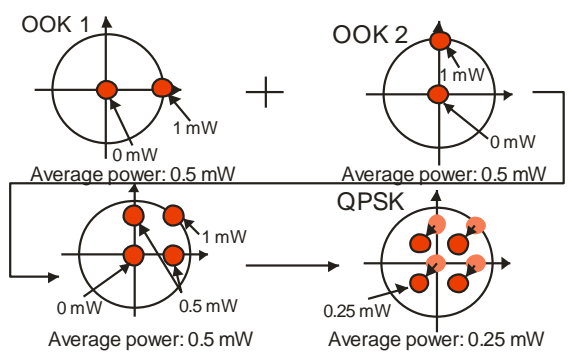

(iv) 16QAM

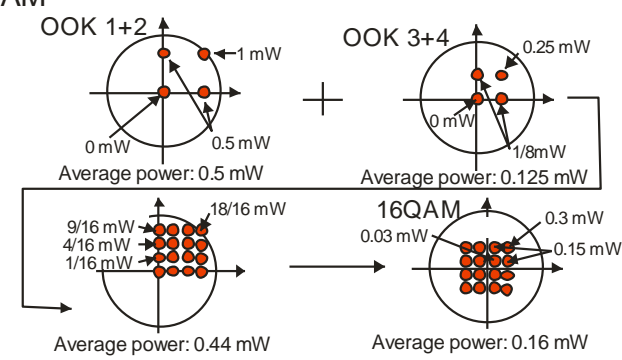

Fig. 2: Schematics of the three schemes under consideration $(a, b, c)$ and constellations showing the principles used to generate QPSK and 16 QAM. The numbers given assume that each laser used has a maximum power of $1 \mathrm{~mW}$.

TABLE 1. Scheme Loss and Modulated Power

\begin{tabular}{|l|l|l|l|l|l|l|}
\hline & \multicolumn{2}{|c|}{$\begin{array}{l}\text { IQ } \\
\text { modulator }\end{array}$} & \multicolumn{2}{l|}{$\begin{array}{l}\text { Opt.-muxing } \\
\text { modulator }\end{array}$} & \multicolumn{2}{l|}{ Our scheme } \\
\hline Format & QPSK & 16 QAM & QPSK & 16QAM & QPSK & 16QAM \\
\hline $\begin{array}{l}\text { Scheme } \\
\text { Loss, dB }\end{array}$ & 3 & 5.5 & 3 & 6 & 6 & 9 \\
\hline $\begin{array}{l}\text { Modulated } \\
\text { Power, } \mathrm{\mu W}\end{array}$ & 500 & 280 & 500 & 250 & 250 & 160 \\
\hline
\end{tabular}

RF power requirements

We consider all modulation devices (modulators, directly-modulated lasers) matched to $50 \Omega$. However, later we also discuss how the RF power could be lowered, e.g., by using matching to another impedance value.

Optical modulators typically require 2 to $8 \mathrm{~V}$ to achieve $\mathrm{V} \pi$. We will consider a value of $3 \mathrm{~V}$ in our analysis, giving a reasonably good expectation of what can be achieved with current technology. Further, as shown in Fig. 2, we consider that all modulators are operated in a push-pull configuration and therefore require two times less RF power when compared to driving a single-electrode modulator.

For directly-modulated lasers, we consider a typical laser used in our proof-of-principle experiments [3] first. Its light-current and voltcurrent characteristics are shown in Fig. 3. In our work, the laser was DC-biased to obtain $35 \mathrm{~mA}$ through the laser. The RF signal was introduced via a Bias Tee with Voltage swing of $\pm 1 \mathrm{~V}$, which (for $50 \Omega$ coupling) corresponds to current modulation of $\pm 20 \mathrm{~mA}$ (and RF power of $10 \mathrm{dBm}$ ). Thus, the directly-modulated laser current varied from $15 \mathrm{~mA}$ to $55 \mathrm{~mA}$, corresponding, Fig. 3 , to $2 \mathrm{~mW}$ to $9 \mathrm{~mW}$.

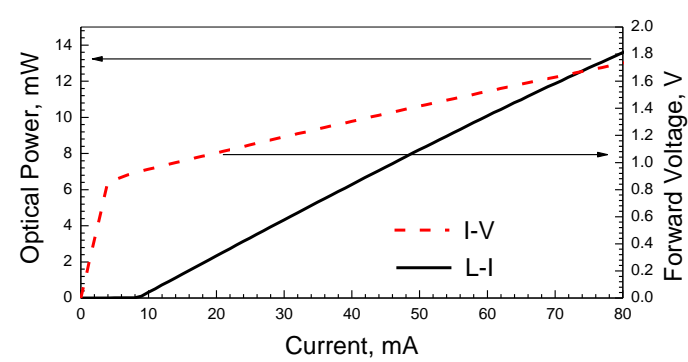

Fig. 3: L-V and I-V curves of the laser used in [3].

For QPSK, the first two schemes require $4 \mathrm{RF}$ streams, each with a swing of $3 \mathrm{~V}$. Our scheme requires only two RF streams (as it is singleended-operated) and $2 \mathrm{~V}$ swing. For $16 \mathrm{QAM}$, the requirements for the IQ modulator remain the same, while the scheme shown in Fig $2 b$ would require $8 \mathrm{RF}$ streams with $3 \mathrm{~V}$ swing each, being two times more as compared to the IQ modulation. Our scheme would require $4 \mathrm{RF}$ streams with $2 \mathrm{~V}$ swing. Thus, considering the lasers used in our experiment (that were not optimized at all for this scheme), this required the least RF power, although this was achieved at the expense of a slightly higher scheme loss and modulated power, Table 1. However, the lasers could be operated at lower impedance, e.g., $25-\Omega$ matching would give a further $3 \mathrm{~dB}$ decrease in the RF power required. Further reduction could be expected by using other laser types, e.g. VCSELs, which typically require 10 $\mathrm{dB}$ less RF power as compared to, e.g., DFB lasers due to their higher slope [5]. This compares to a much smaller expected improvement from the already-well optimized modulator technology, where a voltage reduction to $2 \mathrm{~V}$ is to be expected. Besides lower RF power, our scheme requires a single ended drive only, halving the number of driving signals required.

\section{Conclusions}

Our new QAM synthesis scheme has the potential to significantly reduce the RF power (> $10 \mathrm{~dB}$ ) necessary for the generation of higher order modulation formats, with a minimum penalty in terms of optical loss which is $3 \mathrm{~dB}$ higher for QPSK and $4.5 \mathrm{~dB}$ for 16 QAM when compared to an IQ modulator.

Financial support of EPSRC (Photonics Hyperhighway [EP/I01196X/1] and Fellowship of R. Slavík [EP/K003038/1]) is greatly acknowledged.

\section{References}

[1] A. Chiba et al, Electron. Lett. 46, 220 (2010).

[2] R. Slavik et al, OFC 2013, JTh2A.32

[3] R. Slavík et al, OFC PDP 2013, PDP5.B.5.

[4] S. Mohrdiek, JLT 12, 418 (1994).

[5] P. Gamage et al, PTL 20, 2102 (2008). 